\title{
Addendum to "Quantum Gravity and the Holographic Mass" in View of the 2013 Muonic Proton Charge Radius Measurement
}

\author{
Nassim Haramein ${ }^{\dagger}$ \\ Hawaii Institute for Unified Physics
}

\begin{abstract}
We consider the latest results of the measurement of the charge radius of the proton utilizing laser spectroscopy of muonic hydrogen published in Science on January 25, 2013 by an international team lead by Aldo Antognini and carried out at the Paul Scherrer Institute Proton Accelerator. Given the new charge radius measurement, we compute the proton mass utilizing our generalized holographic approach and find that our result is now within $0.00072 \times 10^{-24} \mathrm{gm}$ of the 2010 -CODATA value of the proton rest mass. Our predicted charge radius is now within $0.00036 \times 10^{-13} \mathrm{~cm}$ and remains within one standard deviation of the new measurement.
\end{abstract}

\section{INTRODUCTION}

On January 25, 2013, the journal Science reported the latest results from Aldo Antognini et al. ${ }^{1}$ on the measurements of the charge and Zemach radii of the proton. The team was able to obtain measurements with 1.7 times more precision than the 2010 muonic hydrogen result ${ }^{2}$ while also confirming the earlier findings. The team reported $0.84087(39) \mathrm{fm}$ for the charge radius which has direct implications to the computations in our original paper, Quantum Gravity and the Holographic Mass. ${ }^{3}$ In this addendum, we recalculate our generalized holographic solution for the mass of the proton and consider our originally predicted charge radius relative to this new result.

\section{HOLOGRAPHIC MASS SOLUTION OF THE PROTON}

In equation (29) of reference [3] (reproduced below), we find the algebraic reduction of our holographic geometric solution derived from the relationship of Planck spherical unit (PSU) volume to horizon surface tiling. Therefore, the latest results from Aldo Antognini et al. ${ }^{1}$ of the muonic charge radius measurement have a critical bearing on our computation of the rest mass of the proton. We replace the 2010 muonic measurement value of the proton charge radius $r_{p}=0.84184(67) \times 10^{-13} \mathrm{~cm}$ in equation (29) of reference [3], with the 2013 reported charge radius $r_{p^{\prime \prime}}=0.84087(39) \times 10^{-13} \mathrm{~cm}$ and obtain a rest mass value of

$$
m_{p^{\prime}}=4 \ell \frac{m_{\ell}}{r_{p^{\prime \prime}}}=1.673349 \times 10^{-24} \mathrm{gm}
$$

\footnotetext{
$\dagger$ Hawaii Institute for Unified Physics - haramein@hiup.org

Copyright (c) 1/31/2013 Nassim Haramein - Pre-Print
} 
where $\ell$ is the Planck length, $m_{\ell}$ is the Planck mass, $r_{p^{\prime \prime}}$ is the latest proton charge radius, and $m_{p^{\prime}}$ is our derived proton rest mass. Thus, our result is now within $0.00072 \times 10^{-24} \mathrm{gm}$ of the 2010-CODATA value for the proton rest mass of $m_{p}=1.672622 \times 10^{-24} \mathrm{gm}$ compared to our earlier result based on the 2010 muonic charge radius measurement ${ }^{2}$ of $m_{p^{\prime}}=1.671421 \times 10^{-24} \mathrm{gm}$ which had a deviation from 2010 -CODATA of $0.0012 \times 10^{-24} \mathrm{gm}$.

In reference [3] equation (30) we go on to predict a precise proton charge radius from the theoretical tenets of our holographic approach utilizing the 2010-CODATA value of the proton rest mass. We reproduce equation (30) below and compare it to the new muonic charge radius result.

$$
r_{p^{\prime}}=4 \ell \frac{m_{\ell}}{m_{p}}=0.841236 \times 10^{-13} \mathrm{~cm}
$$

where $m_{p}$ is the 2010-CODATA rest mass of the proton. Therefore, the 2013 muonic charge radius measurement is now within $0.000366 \times 10^{-13} \mathrm{~cm}$ from our predicted charge radius value compared with a difference of $0.000604 \times 10^{-13} \mathrm{~cm}$ when the 2010 muonic charge radius was utilized. Our predicted proton charge radius is also within one standard deviation of the 2013 muonic charge radius measurement.

\section{CONCLUSION}

In this addendum, we report the consequence of the 2013 muonic measurement of the proton charge radius on our generalized holographic solution to quantum gravity and the proton mass. The more precise charge radius measurement of 2013 provides confirmation of the previous 2010 muonic experimental results. The use of the new radius in our derivation of the proton rest mass generates a value within $0.00072 \times 10^{-24} \mathrm{gm}$ or $0.042 \%$ of the 2010 -CODATA value, where the 2010 muonic measurement resulted in a $0.0012 \times 10^{-24} \mathrm{gm}$ or $0.072 \%$ difference from the reference value. In addition, the new measurement of the proton charge radius is now within $0.00037 \times 10^{-13} \mathrm{~cm}$ of our predicted value, further validating the accuracy of our generalized holographic approach.

\section{REFERENCES}

${ }^{1}$ A. Antognini, et. al., "Proton Structure from the Measurement of 2S-2P Transition Frequencies of Muonic Hydrogen", Science, vol. 339, (25 January 2013).

${ }^{2}$ R. Pohl, A. Antognini, F. Nez, et. al., "The size of the proton", Nature, vol. 466, issue 7303, pp. 213-216 (2010).

${ }^{3}$ N. Haramein, "Quantum Gravity and the Holographic Mass", Copyright, (20 December 2012). 